\title{
Percepción de los jóvenes acerca de sus expectativas a futuro en cuatro instituciones educativas del municipio de Soacha (Cundinamarca, Colombia)
}

\author{
Perception of Young People about their Expectations \\ for the Future in Four Educational Institutions of the \\ Municipality of Soacha (Cundinamarca, Colombia)
}

Liliana I. Badillo Badillo*

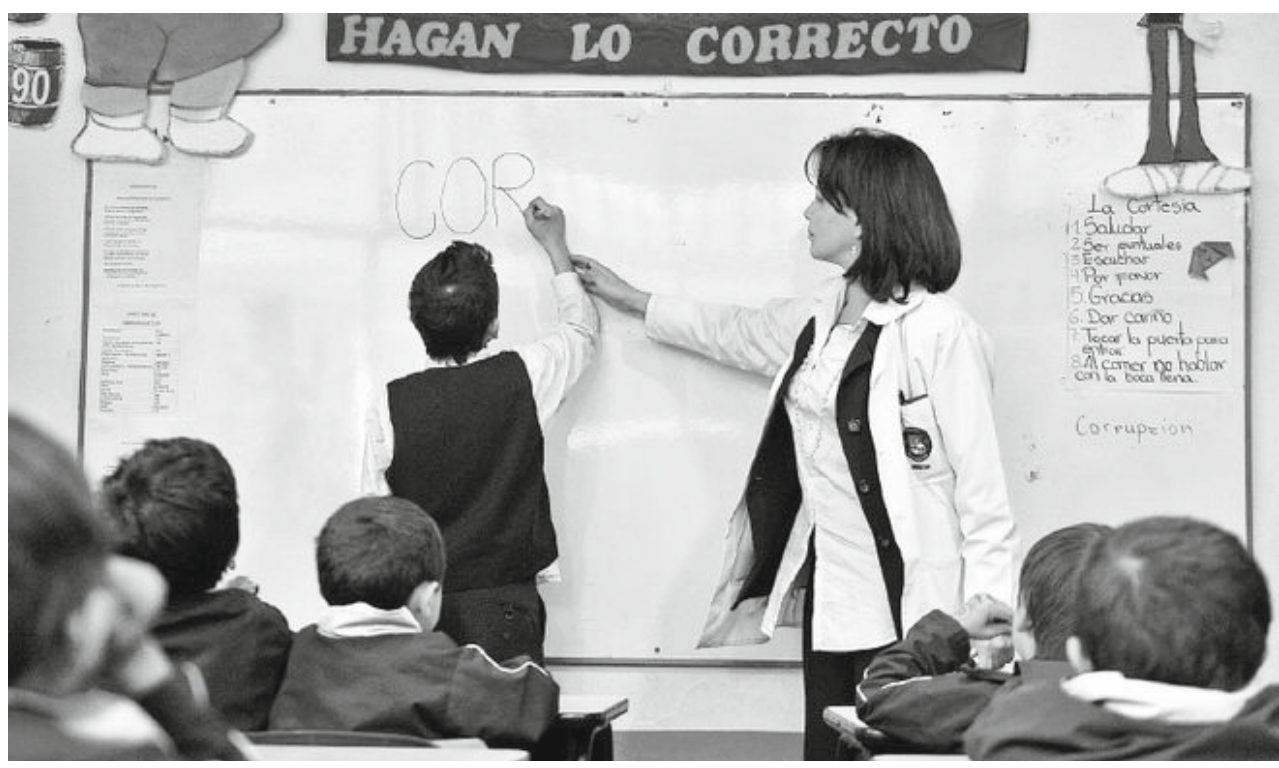

\section{Resumen}

El presente artículo recoge el ejercicio investigativo desde un enfoque cuantitativodescriptivo acerca del análisis de la percepción del área humanista con énfasis en proyecto de vida de tres instituciones educativas de básica y media del municipio de Soacha (Cundinamarca, Colombia). La recolección de datos se realiza a través

Citar este artículo como: Badillo Badillo, L. (2020). Percepción de los jóvenes acerca de sus expectativas a futuro en cuatro instituciones educativas del municipio de Soacha (Cundinamarca, Colombia).

Revista Papeles, 12(23), 86-97.

Fecha de recibido: 1 de marzo del 2020 Fecha de aprobado: mayo 5 de 2020

* Docente licenciada en química y biología de la Universidad Antonio Nariño, especializada en gerencia de instituciones educativas de la Universidad del Tolima, magíster en educación de la Universidad Antonio Nariño. Actualmente se desempeña como directiva docente rectora del Liceo Gregorio Mendel, coordinadora de la I.E. San Mateo y tutora de la Universidad del Tolima. Correo electrónico: libadillo74@uan.edu.co 
del instrumento EFFA (Escala de Expectativas de Futuro en la Adolescencia), con el objetivo de identificar la percepción de los estudiantes de las tres instituciones elegidas acerca de su proyección de vida a mediano plazo. El instrumento EFFA fue diseñado y validado en tres investigaciones previas (Sánchez Sandoval y Verdugo, 2016) y posteriormente adaptado antes de su aplicación en esta investigación. Como resultados de esta investigación, se evidenció que cuanto mayores son las expectativas de futuro de los adolescentes, mayor es también su autoestima, lo que no solo se refleja en su autoconcepto, sino también en la calidad de sus relaciones interpersonales y en su capacidad de vincularse a la sociedad. Lo anterior favorece que los jóvenes con mayores expectativas de futuro aprovechen de mejor manera las oportunidades que les ofrece el entorno para elevar su calidad de vida, aun en los casos en los que no se accede a canales de movilidad social a través del ingreso a instituciones educativas de tercer nivel.

Palabras clave: educación, expectativas, futuro, proyecto de vida.

\begin{abstract}
This article gathers the investigative exercise from a descriptive quantitative approach about the analysis of the perception of the humanistic area with emphasis on the life project of three educational institutions in the municipality of Soacha (Cundinamarca, Colombia). Data collection is carried out through the EFFA instrument (Scale of Expectations for the Future of Adolescence) with the aim of identifying the perception of the students of the four basic and middle educational institutions about their projection of life in the medium term. The EFFA instrument was designed and validated in three previous investigations (Sánchez Sandoval y Verdugo, 2016) and later adapted before its application in this investigation. As a result of this research, it was evidenced that higher future expectations by adolescents produce an increase in their self-esteem, which is not only reflected in their self-concept but in the quality of their interpersonal relationships and their ability to bond with society, too. All of this, allows that young people with higher expectations for the future, take better advantage of the opportunities offered by the environment to improve their quality of life, even in cases in which social mobility channels are not accessed through admission to third level educational institutions.
\end{abstract}

Keywords: Education, Future, Expectations, Life Project.

\title{
Introducción
}

Existen varios referentes para definir al grupo poblacional joven. Así, desde el punto de vista internacional, se reconoce a los jóvenes como el conjunto de la población de entre 15 y 24 años de edad, es decir, un $18 \%$ del total de la población mundial (Organización de las Naciones Unidas [ONU], 2015), quienes son un factor determinante en el cambio social, el desarrollo económico y el progreso técnico. Su imaginación, sus ideales, sus perspectivas y su energía resultan imprescindibles para el desarrollo de las sociedades en las que viven.

De esta manera, se evidencia la necesidad de multiplicar los esfuerzos encaminados a crear y desarrollar políticas y programas que estén

Revista PAPELES • ISSN 0123-0670 • Vol. 12(23) • pp. 86-97 • Enero-junio de 2020 
específicamente destinados a la población juvenil, con especial interés en la identificación de los problemas que les suscitan y con la intención de aprovechar al máximo su potencial, de manera que puedan mejorar su situación socioeconómica actual, así como promover el bienestar de las generaciones futuras.

Para ello, a nivel mundial se han generado iniciativas que garanticen la protección de los jóvenes frente a situaciones peligrosas que ralenticen su desarrollo y frenen su escolarización, que promuevan la educación integral, garantizando la educación primaria de calidad que les permita desempeñar un papel activo en el proceso decisorio en materia social, económica y política, acceder a un trabajo decente, salir de la pobreza, alcanzar un nivel de vida satisfactorio y mejorar su acceso a los sistemas de salud.

La presidencia de la República de Colombia, a través de la Dirección del Sistema Nacional de Juventud Colombia Joven (2013), manifiesta que en el país existen cerca de 12.699.365 jóvenes, quienes constituyen en el eje central en la generación de una agenda para el desarrollo social, cultural, político y económico del país.

A pesar de lo anterior, existe una baja coordinación interinstitucional e intersectorial en la promoción de objetivos conjuntos, con miras a mejorar la calidad de vida de los jóvenes (Betancourth y Cerón, 2017).

Es más, muchas de las iniciativas que se impulsan desde la cooperación internacional, la academia, el sector privado y el sector público tienen escasa participación de los jóvenes, quienes deberían estar llamados a proponer o generar estrategias conjuntas que les beneficien.

Por ello se crea el "pacto", que logra vincular y articular todos los esfuerzos en materia de juventud, que es

un instrumento que materializará la voluntad de todos los sectores que le digan "sí", en la construcción de estrategias, programas, proyectos y desarrollo de acciones dirigidas al mejoramiento de la calidad de vida y la garantía de los derechos de los jóvenes, enmarcados en el trabajo que desde las distintas entidades e instituciones ya se viene adelantando (Presidencia de la República de Colombia, 2015).

El Ministerio de Educación Nacional, en sus planes para la juventud, desea convertir en eje articulador para el proyecto de vida de los jóvenes la educación para el trabajo, motivando las instituciones de básica y media a sentir la necesidad de tener un modelo de educación integral con formación de competencias específicas para el ámbito laboral al que se enfrentan los estudiantes (Ministerio de Educación Nacional [MEN], 2013).

Al respecto, Patricia Martínez (2013) manifiesta que es necesario que haya un "matrimonio" entre educación y empleo. Los jóvenes necesitan desarrollar competencias no solo profesionales, sino laborales que les den la oportunidad de incursionar con éxito en trabajos acordes con sus expectativas de vida.

\section{Marco teórico}

\section{Proyecto de vida}

El ser humano se encuentra en diálogo permanente con el exterior, necesita manifestarse, comunicarse y compartir su vida en la interacción con los demás y el mundo (Forero de Forero y Martínez Acuña, 2001).
Se esperaría que el ser humano pudiera desarrollar y solucionar la compleja gama de situaciones que a lo largo de su vida se le presenta para considerarse productivo $\mathrm{y}$, en consecuencia, poder interactuar en un determinado grupo social. Parte de esas necesidades es el conocimiento los mecanismos por los 
cuales puede planear unos logros a diferentes plazos, alcanzar sus metas de manera escalonada, ordenada y, en consecuencia, una mejora en su calidad de vida que le permita transformar el mundo o simplemente adaptarse a él.

Desde esta perspectiva, puede argumentarse que el proyecto de vida como instrumento

es cimentado en la motivación y la orientación al logro. Para ello se construye en el carácter la autorregulación asumiendo esta organización, cabría considerar la activación de creencias de autoeficacia como una estrategia volitiva capaz de sostener un compromiso más profundo y de mayor calidad con el propio aprendizaje que aquel sostenido mediante la demora de la gratificación. En esta línea, reconociendo las evidencias de que el alumnado con motivación hacia el aprendizaje y el logro presenta unos valores significativamente más altos en rendimiento académico que los alumnos orientados a metas de logro o metas centradas en adquisición de valoración social, cabe señalar también una asociación importante entre el rendimiento académico y la activación de las creencias de auto eficacia (Estévez, Rodríguez, Valle, Regueiro y Piñeiro, 2016, p. 3).

Así, "la unidad de la vida humana es la unidad de un relato de búsqueda" (Macintyre, 2002, p. 65), en la que los únicos criterios de éxito o fracaso de la vida humana como un todo son los criterios de "una búsqueda narrada o susceptible de ser narrada" (Macintyre, 2002, p. 66).

La construcción de proyectos de vida debe estar basada en un concepto de vida y sus dimensiones:

La vida no solamente se limita a fechas y eventos en nuestra historia, sino que es un proceso en el que permanentemente nos vamos conociendo más, vamos adquiriendo conocimientos, habilidades y experiencias, las cuales compartimos con

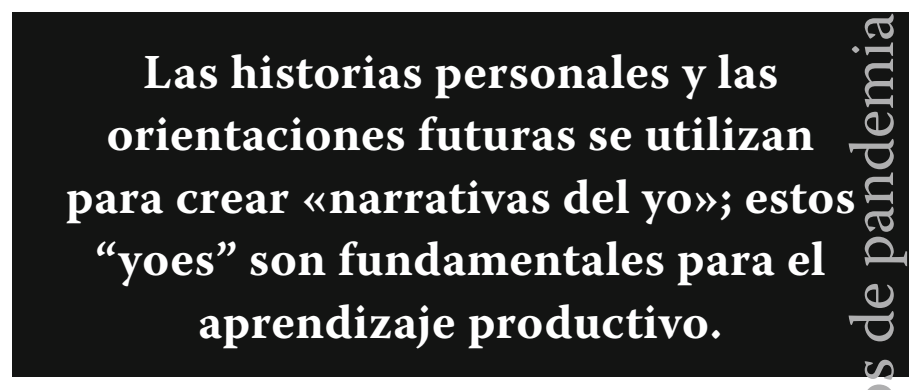

la gente que nos rodea. En este orden de ideas, la sociedad contemporánea con su filosofía facilista e inmediatista de falsos ideales, predominantes del consumo, no representa un punto de referencia sólido para la creación de proyectos de vida en los estudiantes (Roncancio González, 2016, p. 125).

De esta manera, el análisis de la historia personal aparece como un recurso metodológico que a través de las "narrativas del yo" permite la reconstrucción de las trayectorias de aprendizaje y el reconocimiento de los procesos de construcción identitaria que de allí derivan. $\mathrm{Al}$ respecto, Erstad, Gilie y Arnseth (2013, p. 93) manifiestan que

La coherencia entre el aprendizaje, la identidad y la actuación de una persona, enmarcados por un enfoque biográfico que estudia las trayectorias de aprendizaje de las personas durante el transcurso de su vida. Las historias personales y las orientaciones futuras se utilizan para crear «narrativas del yo»; estos "yoes" son fundamentales para el aprendizaje productivo. Con respecto al enfoque basado en «vidas de aprendizaje», la conexión entre el aprendizaje y la identidad es importante porque define la forma en que los distintos estudiantes participan en actividades de aprendizaje en todos sus entornos. El aprendizaje no acaba cuando el alumno sale por la puerta del colegio al final del día.

Por este motivo, es importante planear a futuro, organizar las experiencias, pues "desde el autoconocimiento y el respeto por el ser 
humano se logran generar las oportunidades para que los estudiantes exploren sus talentos y puedan, con el tiempo, decidir sobre sus propios proyectos de vida" (Herrera Vásquez y Gómez Urrutia, 2015, p. 304).

Esto posibilita que se motiven y orienten a sí mismos, lo que requiere valorar la posibilidad que posee cada alumno de comprometerse con su propio proceso orientador, y así lograr las competencias necesarias para la elección y toma de decisiones con responsabilidad y madurez (Berra Bortolotti y Dueñas Fernández, 2015).

Por ello, mientras más posibilidades tengan los sujetos de trabajar sus talentos, hay más probabilidades de incrementar su autonomía y su bienestar subjetivo (Castillo y Contreras, 2014); por ello, es necesario tratar de lograr que el individuo encuentre sentido verdadero a su vida (Redacción Jóvenes, 2013).

Por otra parte, la posibilidad de que todas las personas cultiven sus talentos y habilidades permitiría, a futuro, aumentar su contribución al bienestar de la comunidad (proyección), lo que podría ayudar a resolver problemas locales o nacionales relevantes con recursos propios (autosostenibilidad).

En este marco, una educación fuertemente segregada implica pérdida de parte importante del talento local y una distribución muy inequitativa de las oportunidades para

\section{Mientras más posibilidades tengan los} sujetos de trabajar sus talentos, hay más probabilidades de incrementar su autonomía y su bienestar subjetivo

(Castillo y Contreras, 2014); por ello, es necesario tratar de lograr que el individuo encuentre sentido verdadero a su vida. "agenciar la vida". Como plantea Sen (2000), esto es trabajar desde la propia situación social y con recursos personales para lograr metas que se han definido como importantes desde una jerarquía propia de valores.

Al pensar en el proyecto de vida de estudiantes de básica y media, se deben tener en cuenta variables que podrían afectar dicha proyección.

Hernández y González (2011) estudiaron los efectos diferenciados que los factores económicos, sociales y culturales tienen sobre el logro académico y propusieron un modelo de relación causal entre los constructos o variables latentes: estatus social, cultural y económico (ESCE), nivel económico (NE) y capital cultural escolar (CCE) con el desempeño académico.

La preparación para la vida adulta es en sí una tarea evolutiva adolescente que explica la especial relevancia de la orientación futura (Dreher y Oerter, 1986).

Los intereses de los adolescentes con respecto a su futuro se relacionan con varias dimensiones: educación, trabajo, matrimonio-familia y autopreocupaciones, lo que los hace capaces de establecer objetivos, desarrollar planes y hacer compromisos (Verdugo, Sánchez Sandoval, Melania y Campillo, 2015).

Para lograr que un proyecto de vida notorio evolucione es necesario hacer uso de la inteligencia exitosa, que "es aquella que es verdaderamente importante en la vida, la que se emplea para lograr objetivos importantes y la que muestra quiénes han obtenido éxito en la vida, ya sea según sus patrones personales o los de los demás" (Coll, Mayordomo, Onrubia, Ronchera y Éngel, 2007, p. 3).

Un proyecto de vida debe hacer uso de la creatividad que "tiene un componente personal, que yo cifro en la toma de decisiones, pero también existe una lectura sociocultural. Son estas influencias las que van conformando el modo de ser, pensar y decidir sobre lo que nos sucede en la vida" (De la Torre, 2001, p. 2). 
La creatividad, en tanto que actitud de vida, no es un acto, sino una disposición, y la actitud genera las decisiones creativas en la resolución de problemas; así, "la vida no presenta una predictibilidad de un problema de razonamiento y gran parte de lo que tenemos que aprender en la vida real sobre la resolución de los problemas, sino saber cómo tratar con consecuencias impredecibles o apenas predecibles" (Spear Swerling y Sternberg, 1999, p. 82).

De esta forma, se concreta la relación entre proyecto de vida y educación personalizada, toda vez que uno de los objetivos de esta última "es procurar educar al hombre para que sea capaz de elegir el bien, de ser autónomo tanto a nivel personal como social, con el objeto de concretar su proyecto personal de vida" (Mariz Vásquez y Daura, 2011, p. 27).

De esta manera, es importante crear el vínculo y la acción pertinente para lograrlo. Por ello, "la educación no debe transmitir un caudal de conocimientos sino herramientas para explorar y transformar profundamente la realidad" (Herrera Vásquez y Gómez Urrutia, 2015, p. 10).

\section{Metodología}

Soacha es un municipio considerado la casa de todos, por el crecimiento exponencial de la población en las últimas décadas, fruto de la devaluación de la tierra, el desplazamiento forzado y las soluciones de vivienda ofrecidas en su territorio.

Todos estos fenómenos, unidos a la precaria oferta laboral en el municipio (esto apoyado en el censo de 2003), reportan una tasa de desempleo del $15.6 \%$, pero que podría ser mayor si se tuviese en cuenta el subempleo (Alcaldía municipal de Soacha, 2016).

Esta y otras situaciones hacen del municipio un entorno diverso en todos los aspectos, con grandes zonas de pobreza. En el ámbito educativo se cuenta con 21 colegios oficiales y 185 colegios privados oficialmente reconocidos, que están distribuidos en 6 comunas (Secretaría de educación de Soacha, 2018).

Para la presente muestra se seleccionaron 3 instituciones educativas: dos oficiales, la Institución Educativa San Mateo (IESMA) y la Institución Educativa Compartir; y una institución privada, el Liceo Gregorio Mendel.

Las tres instituciones tienen características socioeconómicas similares y cuyos proyectos educativos institucionales buscan desarrollar en sus estudiantes un programa de proyecto de vida, gracias a la proyección dada por el equipo docente en las diferentes áreas de conocimiento.

Las instituciones seleccionadas comparten la atención de estudiantes entre los 11 y los 24 años para la educación básica y media en jornada diurna. Cada una de ellas varía la cantidad de estudiantes que atiende; por ello, la muestra en las instituciones oficiales corresponde al $10 \%$ de estudiantes y en las privadas el 50\% de la población, escogidos de manera aleatoria, cuya única condición es pertenecer como estudiante regular a la institución seleccionada.

La selección de la muestra obedece a los siguientes criterios: escolarizado en los niveles de básica o media, jornada diurna, proyecto educativo institucional que contemple proyecto de vida como objetivo de formación.

La presente investigación es de corte cuantitativo-descriptivo basado en un análisis estadístico. En este sentido, esta investigación se puede abordar desde la generalidad teórica, en la que los planteamientos de corte cuantitativo se dirigen a:

1. Explorar fenómenos, eventos, comunidades, hechos y conceptos o variables (su esencia es exploratoria); 
2. Describirlos (su naturaleza es descriptiva);

3. Vincularlos (su esencia es correlativa); y

4. Considerar los efectos de unos en otros (su naturaleza es causal [Hernández Sampieri, Fernández Collado y Baptista Lucio, 2014]).

Se ofrece una idea completa del fenómeno cuando se requiere delinear las características específicas descubiertas por las investigaciones exploratorias. Esta descripción se realiza usando métodos cuantitativos que tienen como función esencial medir (de la forma más precisa posible) las características, propiedades, dimensiones o componentes del fenómeno en estudio (Díaz Nárvaez y Calzadilla Nuñez, 2016).

En esta investigación no existe la manipulación de variables o la intención de búsqueda de la causa-efecto con relación al fenómeno, su diseño descriptivo describe lo que existe, determina la frecuencia en que este hecho ocurre y clasifica la información (Souza, Driessnack y Costa Mendes, 2007).

\section{Resultados}

Los resultados de la presente investigación se obtuvieron con el instrumento estandarizado EFFA, relacionado con las expectativas a futuro y los sueños a futuro de los grupos escolares estudiados. Este instrumento fue aplicado a los estudiantes de las tres instituciones educativas participantes, por lo que se realizó un análisis comparado acerca de las percepciones de los encuestados en estas instituciones, en las que el proyecto de vida es un objetivo de la acción pedagógica.

\section{EFFA y expectativas de futuro}

Se encuestaron 173 estudiantes escolarizados en básica y media, diurna, entre los 10 y los 24 años de tres instituciones del municipio de Soacha, las cuales comparten como objetivo en su proyecto educativo institucional el desarrollo del proyecto de vida. Se realizó la prueba de fiabilidad al instrumento aplicado a través del coeficiente alfa de Cronbach, con una puntuación de 0.82 , lo cual evidencia la coherencia interna del mismo.

El instrumento EFFA corresponde a 14 preguntas organizadas en 4 factores: el factor I corresponde a expectativas económicolaborales, el factor II trata de expectativas académicas, el factor III sobre las expectativas de bienestar personal y el factor IV de expectativas familiares. Se contestan las preguntas en una escala de 5 posibles respuestas.
La puntuación de cada ítem indica el grado de probabilidad de que ocurran o no ciertas cosas en su futuro, con el siguiente formato de respuesta: 1 = "estoy seguro/a de que no

Tabla 1. Instrumento EFFA

\begin{tabular}{|c|c|c|c|c|}
\hline Pregunta & 1 & 2 & 3 & 4 \\
\hline Terminaré el bachillerato & & & & \\
\hline Encontraré trabajo & & & & \\
\hline Tendré un carro & & & & \\
\hline Terminaré una carrera técnica & & & & \\
\hline $\begin{array}{l}\text { Encontraré un trabajo que me } \\
\text { guste }\end{array}$ & & & & \\
\hline Tendré una vida familiar feliz & & & & \\
\hline Seré respetado por todos & & & & \\
\hline Me sentiré seguro & & & & \\
\hline Tendré una casa & & & & \\
\hline $\begin{array}{l}\text { Realizaré estudios } \\
\text { universitarios }\end{array}$ & & & & \\
\hline $\begin{array}{l}\text { Encontraré un trabajo bien } \\
\text { pago }\end{array}$ & & & & \\
\hline $\begin{array}{l}\text { Encontraré una persona con } \\
\text { quien formar una pareja feliz } \\
\text { y estable }\end{array}$ & & & & \\
\hline Seré feliz & & & & \\
\hline Tendré hijos & & & & \\
\hline
\end{tabular}


ocurrirán"; 2 = "es difícil que ocurra"; 3 = "puede que sí/puede que no"; 4 = "probablemente ocurra"; y 5 = "estoy seguro/a de que ocurrirá"; tal y como se observa en la tabla 1:

\section{Análisis comparativo a partir del instrumento EFFA}

Con el ánimo de establecer una comparación entre la percepción de los estudiantes de las diferentes instituciones educativas, se realizó la prueba estadística de $\mathrm{H}$ de Kruskal-Wallis, la cual compara muestras independientes, como es el caso de las instituciones educativas estudiadas (como se muestra en la tabla 2).

De esta manera, fueron comparados los cuatro factores con los cuales se categoriza el instrumento EFFA en relación con las respuestas de los estudiantes de las diferentes instituciones educativas.
De acuerdo con los resultados, se observa una diferencia estadísticamente significativa (sig., asintótica $>0.05$ ) en los cuatro factores, lo cual significa una alta heterogeneidad en las respuestas de los estudiantes entre las diferentes instituciones educativas.

Asimismo, es importante resaltar que la percepción favorable de los estudiantes en relación a las expectativas académicas (factor II) es significativamente superior en el Liceo Gregorio Mendel, en comparación con las otras instituciones educativas (rango promedio = 80.98); mientras que en los factores asociados a las expectativas económico-laborales (factor I), bienestar personal (factor III) y expectativas familiares (factor IV), los estudiantes de la Institución Educativa San Mateo evidencian una percepción más favorable en comparación con las demás instituciones educativas, con rangos promedios de $81.27,81.73$ y 78.92 , respectivamente.

Tabla 2. Análisis comparativo entre los cuatro factores del instrumento EFFA a través de la prueba H de Kruskal-Wallis

\begin{tabular}{|c|c|c|c|c|c|c|}
\hline Factor & Institución educativa & $\mathbf{N}$ & $\begin{array}{c}\text { Rango } \\
\text { promedio }\end{array}$ & $\begin{array}{c}\text { H de } \\
\text { KruskalWallis }\end{array}$ & gl & $\begin{array}{l}\text { Sig. } \\
\text { asintótica }\end{array}$ \\
\hline \multirow{4}{*}{$\begin{array}{l}\text { I: expectativas } \\
\text { económico-laborales }\end{array}$} & Liceo Gregorio Mendel & 33 & 80,98 & \multirow{4}{*}{5,584} & \multirow{4}{*}{2} & \multirow{4}{*}{0,061} \\
\hline & San Mateo & 76 & 81,27 & & & \\
\hline & Compartir & 42 & 62,55 & & & \\
\hline & Total & 151 & & & & \\
\hline \multirow{4}{*}{$\begin{array}{l}\text { II: expectativas } \\
\text { académicas }\end{array}$} & Liceo Gregorio Mendel & 33 & 80,80 & \multirow{4}{*}{3,129} & \multirow{4}{*}{2} & \multirow{4}{*}{0,209} \\
\hline & San Mateo & 76 & 79,28 & & & \\
\hline & Compartir & 42 & 66,30 & & & \\
\hline & Total & 151 & & & & \\
\hline \multirow{4}{*}{ III: bienestar personal } & Liceo Gregorio Mendel & 33 & 68,45 & \multirow{4}{*}{2,792} & \multirow{4}{*}{2} & \multirow{4}{*}{0,248} \\
\hline & San Mateo & 76 & 81,73 & & & \\
\hline & Compartir & 42 & 71,56 & & & \\
\hline & Total & 151 & & & & \\
\hline \multirow{4}{*}{$\begin{array}{l}\text { IV: expectativas } \\
\text { familiares }\end{array}$} & Liceo Gregorio Mendel & 33 & 74,74 & \multirow{4}{*}{0,784} & \multirow{4}{*}{2} & \multirow{4}{*}{0,676} \\
\hline & San Mateo & 76 & 78,92 & & & \\
\hline & Compartir & 42 & 71,70 & & & \\
\hline & Total & 151 & & & & \\
\hline
\end{tabular}

Fuente: elaboración propia 


\section{Análisis de las expectativas}

Con base en el análisis comparativo de los cuatro factores del instrumenteo EEFA descrito anteriormente, se hace posible el siguiente análisis de las expectativas a futuro de los jóvenes escolarizados en básica media de las tres instituciones educativas oficiales y privadas del municipio de Soacha (San Mateo, Compartir y Liceo Gregorio Mendel, respectivamente).

\section{Expectativas}

La adolescencia es una etapa clave para revisar los procesos de formación de la niñez y forjar estructuras sólidas en la consolidación de proyectos de vida; por ello, el instrumento EFFA permite observar, desde la perspectiva de los adolescentes, cómo visualizan su propio futuro.

Las instituciones educativas observadas tienen la particularidad de incluir el diseño del proyecto de vida en sus currículos, no solo en las áreas del conocimiento, sino también de manera transversal, como meta de desarrollo del área humanista.

Las tres instituciones en los niveles de básica y media tienen estudiantes en un rango de edad de los 11 a los 21 años, de los estratos 1 al 4, y con un ingreso familiar no superior a los 4 salarios mínimos mensuales.

El instrumento tiene cuatro factores. El factor I que contempla expectativas laborales y económicas mostró que los jóvenes se manifiestan optimistas frente a la consecución de un trabajo estable, bien remunerado y que sea de su agrado.

Pese a esto, existen diferencias entre las instituciones, entre las que se destaca que los estudiantes de la institución educativa San Mateo consideran que, aunque es difícil conseguir trabajo, este les agradará y será bien remunerado. Casi la totalidad aspira a tener casa propia; totalidad que disminuye en cuanto a la adquisición de carro. Para los jóvenes de la Institución Educativa Compartir, las tres condiciones serán alcanzables, pero se ve con dificultad la tenencia de vivienda y solo un poco más de la mitad consideran tener auto; mientras que en el Liceo Gregorio Mendel las dos primeras serán alcanzables, pero la remuneración a la labor no será considerada adecuada. Casi todos aspiran a tener vivienda propia, pero disminuye a menos de tres cuartas partes a la consecución de vehículo.

En cuanto al factor II, que revisa el futuro académico deseado, un porcentaje superior al 90\% en el Liceo Gregorio Mendel y la Institución Educativa San Mateo aspiran a terminar el bachillerato, la educación técnica e ir a la universidad, en tanto los jóvenes de la Institución Educativa Compartir desean terminar el bachillerato, menos de tres cuartas partes de los estudiantes aspiran a los estudios técnicos, y un porcentaje menor a los universitarios.

El factor III sobre expectativas de bienestar personal, respeto, seguridad y felicidad también muestra diferencias en las poblaciones estudiadas. La proporción de estudiantes que no atribuyen un alto valor a la seguridad corresponde a menos de la tercera parte de la población en el Liceo Gregorio Mendel y en la Institución Educativa San Mateo, que es mucho más baja en la Institución Educativa Compartir.

El factor IV, en referencia a la familia, es el que se analiza como el más bajo, ya que el concepto de familia apenas registra el 60\% de valoración positiva, lo que está en correspondencia con el hecho que la estabilidad de pareja alcance el $50 \%$ de valoración.

Por su parte, mientras menos de la mitad de los estudiantes de los colegios San Mateo y Compartir desean tener hijos, en el caso del Liceo Gregorio Mendel este porcentaje aumenta al $60 \%$. 


\section{Conclusiones}

La investigación muestra que cuanto mayores son las expectativas de futuro de los adolescentes, mayor es también su autoestima (Sánchez Sandoval y Verdugo, 2016), lo que se refleja en el autoconcepto, y esto a su vez tiene un efecto tranquilizador en los demás, lo cual permite unas buenas relaciones interpersonales o afectotimia (Alonso Palacio et al., 2007), que redunda en los beneficios esperados a futuro.

Además, el acceso al trabajo adquiere valor constitutivo de su identidad y construcción de su subjetividad, el cual es uno de los pilares (junto al hallazgo del objeto amoroso) que da cuenta de la resolución adolescente, en parte, por la autonomía económica que implica (De Lucca y Petriz, 2006). Sin duda, se podría afirmar que una expectativa ambiciosa garantiza logros alcanzables a futuro.
En relación al análisis comparativo de la percepción de los estudiantes de las diferentes instituciones educativas y a cada uno de los cuatro factores analizados a través del instrumento EFFA, sobresale la percepción favorable de los estudiantes del Liceo Gregorio Mendel en relación con las expectativas académicas, lo cual ratifica los esfuerzos que desde la modalidad de educación personalizada se adelantan en el dicho liceo, con el fin de favorecer oportunidades en los estudiantes que les posibilite una mejor calidad de vida.

En relación con los otros tres factores, se evidencia una percepción más favorable por parte de los estudiantes de la Institución Educativa San Mateo en comparación con las otras instituciones educativas.

\section{Referencias}

Alcaldía municipal de Soacha. (2016). Alcaldía de Soacha-Cundinamarca. Soacha, Colombia: Alcaldía municipal. Recuperado de http://www.soacha-cundinamarca.gov.co/ informacion_general.shtml\#identificacion

Alonso Palacio, L. M., Murcia Gandara, G., Murcia Gandara, J., Herrera Pertuz, D., Gómez Guzman, D., Comas Vargas, M. y Ariza Theran, P. (2007). Autoestima y relaciones interpersonales en jóvenes estudiantes de primer semestre de la División Salud de la Universidad del Norte, Barranquilla (Colombia). Salud Uninorte, 32-42.

Berra Bortolotti, M. J. y Dueñas Fernandez, R. (2015). Carpeta de proyecto de vida: una herramienta de evaluacion. Remo, 42-49.

Betancourth Zambrano, S. y Cerón Acosta, J. (2017). Adolescentes creando su proyecto de vida profesional desde el modelo DPC. Revista Virtual Universidad Católica del Norte, 50, 22-41.
Castillo, J. y Contreras, D. (2014). El Papel de la Educación en la formación del Bienestar Subjetivo para el Desarrollo Humano. Una revisión al caso chileno. Santiago de Chile: Programa de las Naciones Unidas para el Desarrollo - Fondo de las Naciones Unidas para la Infancia.

Coll, C., Mayordomo, R., Onrubia, J., Ronchera, M. J. y Éngel, A. (2007). La "inteligencia exitosa" según R. J. Sternberg. Barcelona, España: Universitat de Barcelona. Recuperado de http:// s623319320.web-inicial.es/wp-content/ uploads/2019/03/Lectura-inteligenciaexitosa-1.pdf

De la Torre, S. (2001). Conversando con Robert J Sternberg sobre creatividad. Barcelona, España: Universitat de Barcelona. Recuperado de http://www.ub.edu/sentipensar/ pdf/saturnino/conversando_con_robert_ sobre_creatividad.pdf 
De Lucca, G. y Petriz, G. (2006). Crisis de las significaciones sociales, el adolescente y su proyecto de futuro laboral. Orientación y sociedad, 1-7.

Díaz Nárvaez, V. P. y Calzadilla Nuñez, A. (2016). Tipos de investigación y productividad científica en las ciencias de la salud. Ciencias de la salud, 115-121.

Dirección del Sistema nacional de juventud. (2013). Colombia joven. Bogotá: Consejería presidencial para la juventud. Recuperado de http://www.colombiajoven. gov.co/colombiajoven/queescolombiajoven

Dreher, E. y Oerter, R. (1986). Children's and Adolescents' Conceptions of Adulthood: The Changing View of a Crucial Developmental Task. Munich: Springer Berlin Heidelberg.

Erstad, O., Gilie, O. y Arnseth, H. C. (2013). Vidas de aprendizaje conectadas: jóvenes digitales en espacios escolares y comunitarios. Comunicar, 89-98.

Estevez, I., Rodríguez, S., Valle, A., Regueiro, B. y Piñeiro, I. (2016). Incidencia de las metas académicas del alumnado de secundaria en su gestión motivacional. Aula abierta, 83 -90 .

Forero de Forero, A. y Martínez Acuña, M. (2001). Formación integral y educación personalizada. Chía, Colombia: Universidad de La Sabana. Recuperado de https://www.unisabana.edu.co/fileadmin/Archivos_de_usuario/Documentos/ Documentos_la_Universidad/Docs_Institucionales/8._Formacion_Integral_y_Educacion_Personalizada.pdf

Hernández Sampieri, R., Fernández Collado, C. y Baptista Lucio, M. (2014). Metodología de la investigación. México: McGraw Hill.

Hernández, E. y Gonzalez, M. (2011). Modelo de ecuación estructural que evalúa las relaciones entre el estatus cultural y económico del estudiante y el logro educativo. Revista electrónica de investigación educativa, 188-203.
Herrera Vásquez, L. y Gómez Urrutia, V. (2015). Disonancias entre la eduación actual y el desarrollo humano. Una discusión clave. Páginas sobre educación, 1-23.

Macintyre, A. (2002). Tras la virtud. Barcelona: Crítica.

Mariz Vásquez, S. y Daura, F. T. (2011). La educación personalizada. Algunas precisiones en torno al concepto. Revista Panamericana de Pedagogía, 17-37.

Ministerio de Educación Nacional (MEN). (2009). El ideal educativo del nuevo siglo. $A l$ tablero, 52, Septiembre-Octubre, 16-17.

Ministerio de Educación Nacional (MEN). (2013). Eduación inclusiva e intercultural. Bogotá: MEN-Centro virtual de noticias de la educación. Recuperado de https:// www.mineducacion.gov.co/1759/w3-article-340146.html?_noredirect=1

Organización de las Naciones Unidas (ONU). (2015). Juventud. Nueva York: Naciones Unidas. Recuperado de https://www.un.org/ es/sections/issues-depth/youth-0/index. html

Presidencia de la República de Colombia. (16 de Septiembre de 2015). Sistema Nacional de Juventud. Bogotá: Consejería presidencial para la juventud-Presidencia de la República. Recuperado de http://www.colombiajoven. gov.co/participa/snj

Redacción Jóvenes. (7 de agosto de 2013). Educación personalizada; alternativa de aprendizaje. Vanguardia. Recuperado de https://www.vanguardia.com/entreteni miento/jovenes/educacion-personalizadaalternativa-de-aprendizaje-favl219639

Roncancio González, H. (2016). Trayectos vitales en la deserción estudiantil: re-pensar la construcción de proyectos de vida en la formación superior de la institución universitaria Envigado. Revista del Instituto de Estudios en Educación, Universidad del Norte, 1-14.

Sánchez Sandoval, Y. y Verdugo, L. (2016). Desarrollo y validación de la Escala de 
Expectativas de Futuro en la Adolescencia (EEFA). Anales de Psicología, 1695-2294.

Secretaría de Educación de Soacha. (2018). Información Día E 2018. Soacha, Colombia: Soacha educativa. Recuperado de https:// www.soachaeducativa.edu.co/index.php/ component/k2/item/827-informacion-diae-2018

Sen, A. (2000). Desarrollo y libertad. Buenos aires: Planeta.

Souza, V., Driessnack, M. y Costa Mendes, I. A. (2007). Revisión de diseños de investigación resaltantes para enfermería. Parte
1: diseños de investigación cuantitativa. Latinoamericana de Enfermeria, 15-21.

Spear Swerling, L. y Sternberg, R. (1999). Enseñar a pensar. Madrid: Santillana.

Verdugo, L., Sanchez Sandoval, Y., Melania, C. y Campillo, E. (2015). Evaluación de la adaptación escolar de chicos y chicas en acogimiento residencial en centros de la bahía de Cádiz. En Asociación Interuniversitaria de Investigación Pedagógica (AIDIPE [Ed.]), Investigar con y para la sociedad (pp. 489-500). Cádiz, España: AIDIPE 